\title{
THE PATTERN OF AGEING OF THE ARTICULAR CARTILAGE OF THE ELBOW JOINT
}

\author{
John W. Goodfellow and Peter G. Bullough, Oxford, England \\ From the Nuffield Department of Orthopaedic Surgery, University of Oxford
}

Articular cartilage, in common with other tissues of the body, is known to alter in its composition and its physical properties as it grows old. The bluish-white translucency of youth gives way to a yellowish opacity, and surface defects appear here and there.

Something is known of the biochemical and histological changes which accompany these visible signs of ageing. Changes occur in the content of chondroitin sulphate and in its staining qualities (Hirsch 1944), the water content diminishes (Eichelberger, Akeson and Roma 1958), and the orientation of the collagen fibres is said to alter and become predominantly vertical (Little, Pimm and Trueta 1958). The older the tissue the more brittle and rigid it becomes, because, it is believed, of the altered ratio of mucopolysaccharide to collagen (Barnett, Davies and MacConaill 1961).

That these changes and many others are known to occur generally in articular cartilage throughout the body is not enough to explain the localisation of the severer forms of degeneration to particular joints or parts of joints.

Most authors ascribe localised failure of articular cartilage to excessive wear and tear. Among the factors which might increase wear and tear, weight bearing and the transmission of large shearing forces have been regarded as the most important (Ekholm 1956). Within the weight-bearing joints " pressure areas" have been described to account for the observed degenerative lesions (Lloyd-Roberts 1955).

On the other hand, there is evidence that degenerative changes occur in those areas of cartilage which are not subject to the alternating pressures of weight bearing. Harrison, Schajowicz and Trueta (1953) found the first visible degenerative changes in the human hip joint in the non-pressure areas. Perhaps cartilage may deteriorate from too little use rather than too much.

The question remains open: why do some joints degenerate and others not? And why do particular areas of cartilage within those joints degenerate and other parts within the same joint survive?

These questions cannot be answered until it is known which joints or parts of joints do usually degenerate. The clinician knows the joints which commonly become stiff and painful, but because severe cartilage degeneration can occur without causing symptoms this knowledge is of little use in detecting the real incidence. Radiological surveys are of little value because a normal radiograph by no means excludes the presence of degenerate cartilage. There have been few comprehensive studies of age changes in human joints (Bennett, Waine and Bauer 1942) and the state of affairs to be expected in particular joints at particular ages is for the most part unknown.

This paper attempts an answer to the questions posed above from a consideration of the process of ageing observed in the human elbow joint. The argument proceeds in three stages: 1) a description is given of the pattern of degeneration observed from maturity to old age ; 2) the mechanical environment of the cartilage in each part of the articulation is discussed; and 3) an explanation of the pattern of degeneration is attempted by correlating it with what we know of the mechanics of the joint.

The elbow joint is a peculiarly suitable laboratory for the study of the response of cartilage to its mechanical circumstances, because within one synovial cavity there are to be found areas of cartilage subject to a variety of mechanical circumstances. 


\section{PATTERN OF DEGENERATION OF ARTICULAR CARTILAGE IN THE ELBOW}

The radio-humeral joint-Figure 1 shows a collection of radial heads from twenty-eight necropsy subjects. They are arranged in columns according to age. Those in the first column from young adult subjects (eighteen to thirty years) appear at first sight immaculate. Nevertheless, early degenerative changes are in fact to be seen in all of them and will be discussed shortly.

In the second column the changes of middle age can be seen. The articular cartilage on the rim of the radial head is roughened and irregular in all, but the cartilage elsewhere remains smooth. The right radial head of the forty-year-old man at the top of this column has a small ulcer on the postero-medial part of the rim, with bone exposed in its base. This is the earliest indication of the severe degeneration which occurs at this site in succeeding decades.
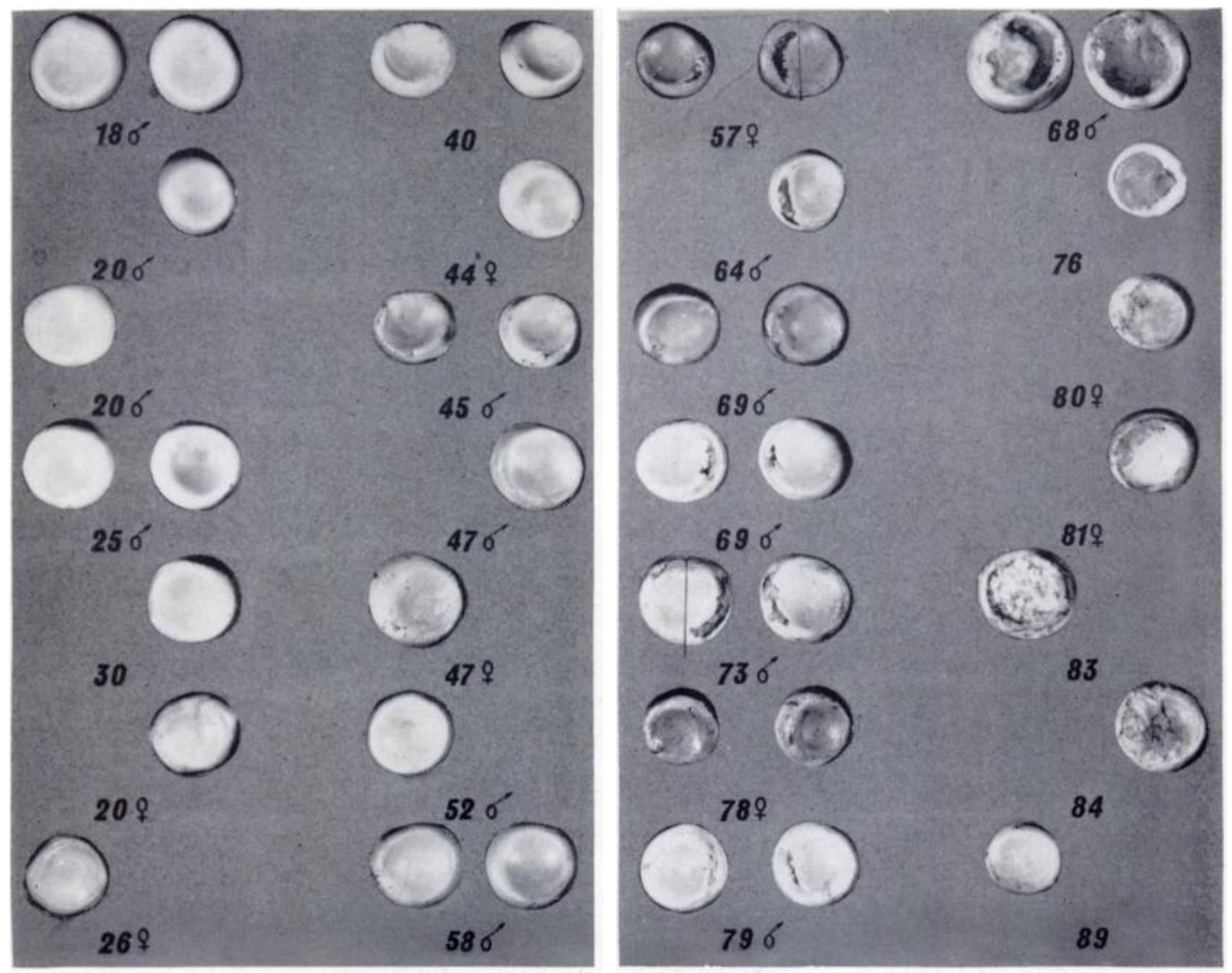

Fig. 1

Radial heads showing progressive degenerative changes with increasing age.

In the third and fourth columns the progress of the changes into old age is shown. The third column shows the localisation of the most severe cartilage changes to the postero-medial part of the rim of the radial head. In every case the cartilage was eroded in this area and the subchondral bone was exposed. The fourth column shows the ultimate destruction of the joint surface which occurs in very old age. In five of the seven subjects the articular surface was devoid of cartilage or covered only by ragged remnants.

The process, which this series of joints shows, starts in early adult life. Chondromalacia of the rim of the head of the radius was present even in the youngest of our subjects. Figure 2 shows the joint surface in an eighteen-year-old man and is typical. The superficial layer of 


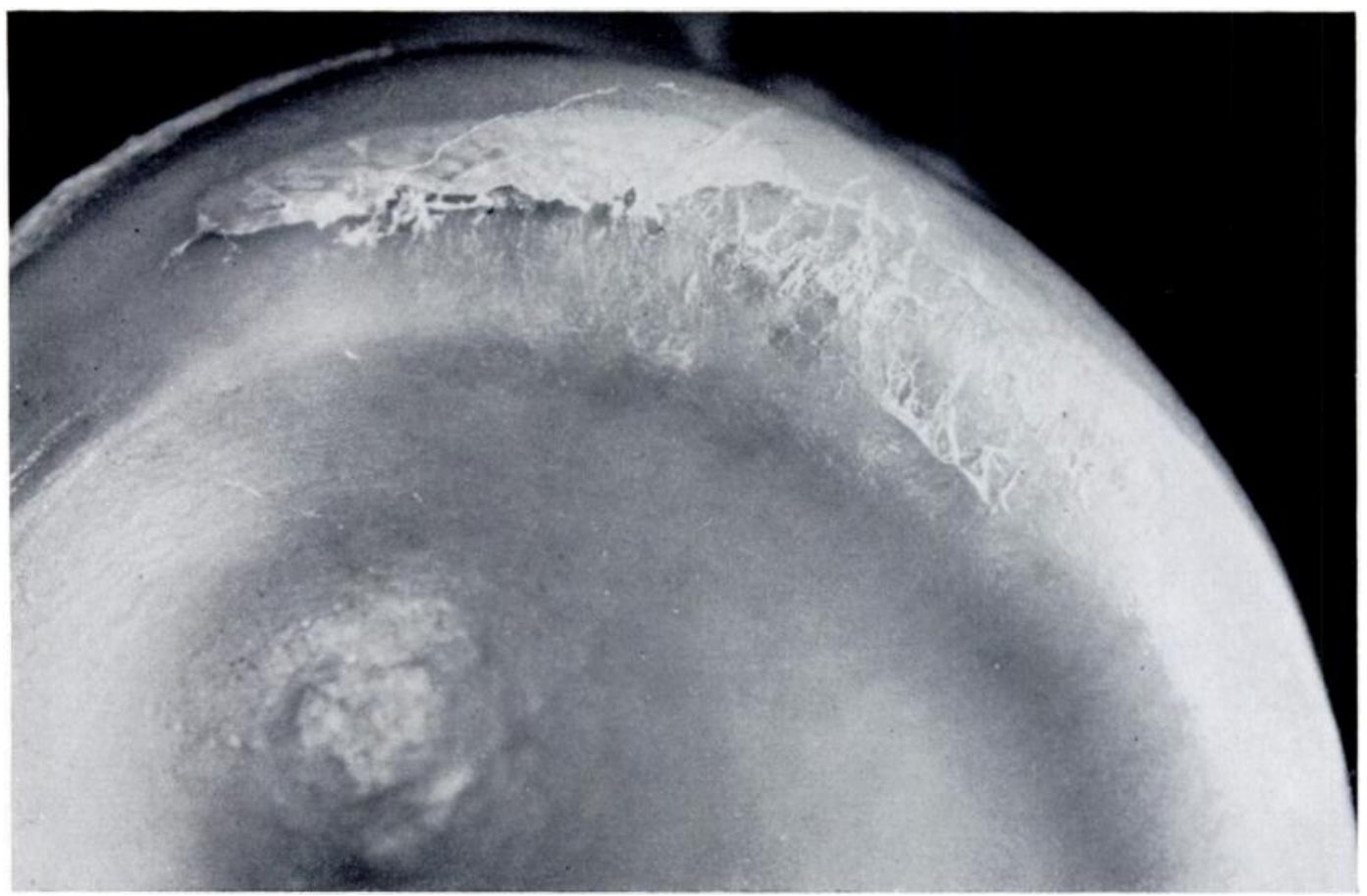

FIG. 2

Part of the radial head of an 18-year-old man showing chondromalacia of the rim. $(\times 10$. $)$

the cartilage is partly detached and the surface of the rim is finely irregular. In several subjects this detachment of the surface layers of the cartilage extended all round the head so that a sort of fringe resulted. The changes are strictly limited to the rim of the head and, indeed, were never observed in youth to affect either the central depression which articulates with the capitulum or the triangular medial facet which articulates with the ulna. In Figure 3 the areas of roughened cartilage have been defined by dipping the specimen in indian ink and then washing it.

When we examined the articular surface of the capitulum we found a mirror image of the pattern of age changes in the radial head. A linear ulcer was constantly present on the posterior part of the crest which separates the trochlea from the capitulum, opposite the postero-medial ulcer on the head of the radius (Fig. 4). The specimens in Figures 5 to 9 show this well; Figures 7, 8 and 9 (subjects aged fifty-seven, eighty-four and seventy-six respectively) show the final stages of degeneration, when the cartilage

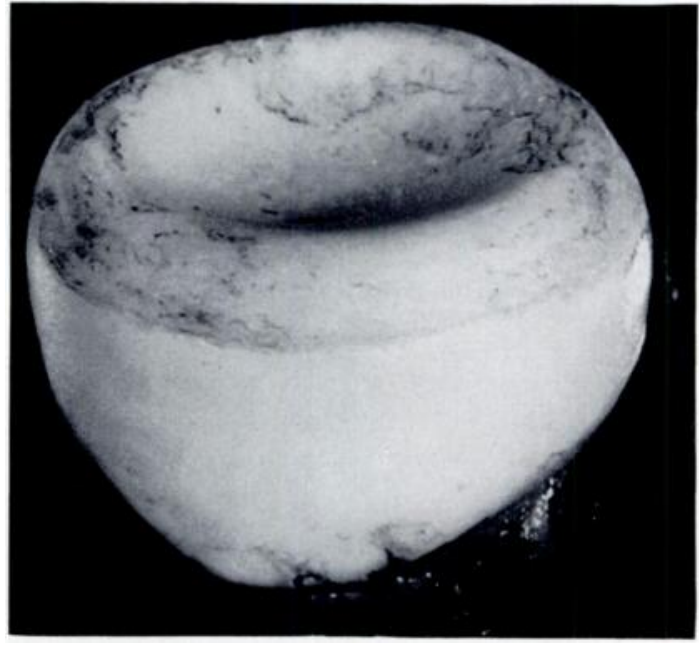

FIG. 3

A radial head which was dipped in indian ink to define the roughened areas. Note the absence of any roughening of the medial triangular facet.

on the capitulum and the opposed central depression of the radial head are destroyed in old age. The humero-ulnar joint-In contrast the cartilage of the humero-ulnar articulation shows no such pattern of degeneration. In older subjects, it is true, the cartilage is often scored in the line of movement (Fig. 8), but these surface grooves never expose the underlying bone.

VOL. 49 B, NO. 1, FEBRUARY 1967 


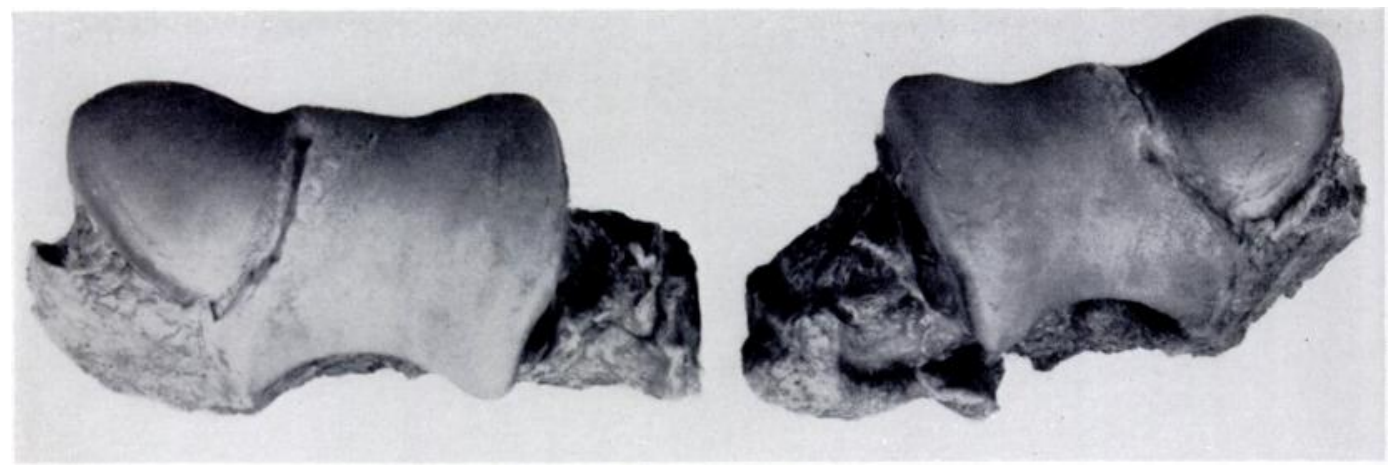

FIG. 4

Symmetrical ulceration of the posterior part of the crest which separates the capitulum from the trochlea. Right and left joints of a man aged 69 years.

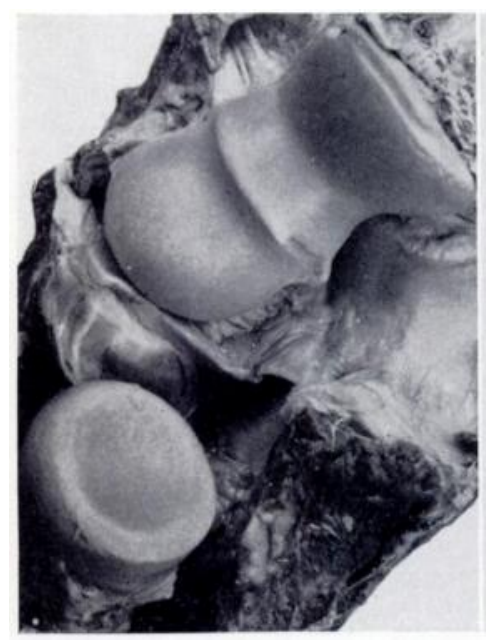

Fig. 5

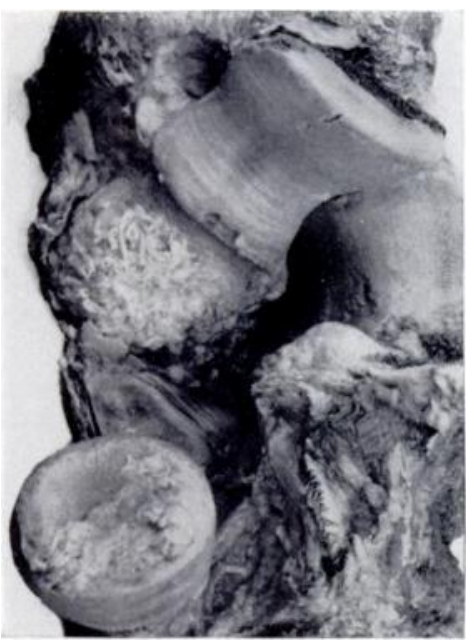

FIG. 8

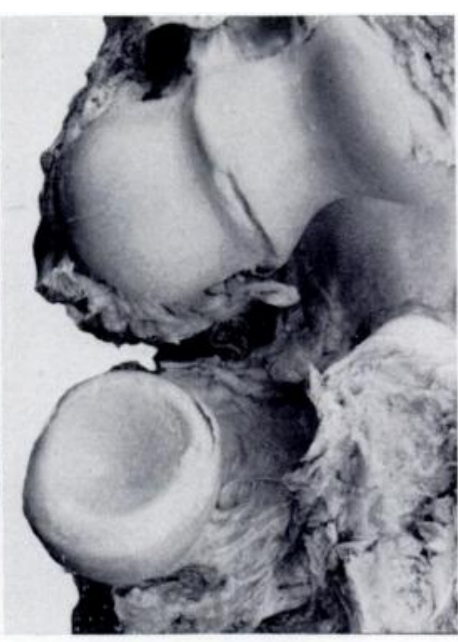

Fig. 6

FIGs. 5 To 9

Figure 5-The displayed joint of a subject aged 30 years.

Figure 6-Subject aged 47 years.

Figure 7-Subject aged 57 years.

Figure 8-Subject aged 84 years.

Figure 9-Subject aged 76 years.

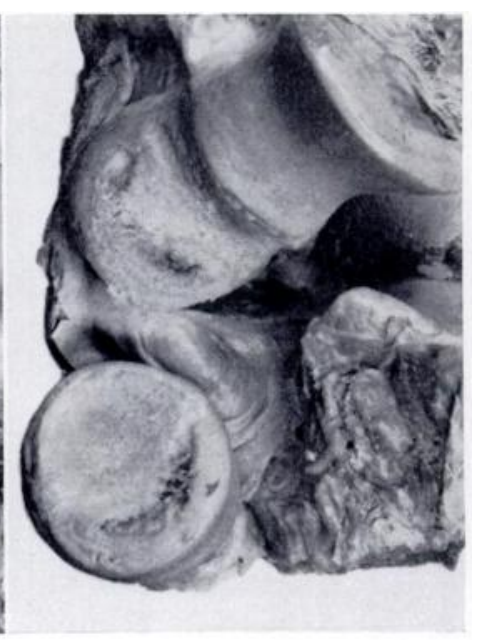

FIG. 7

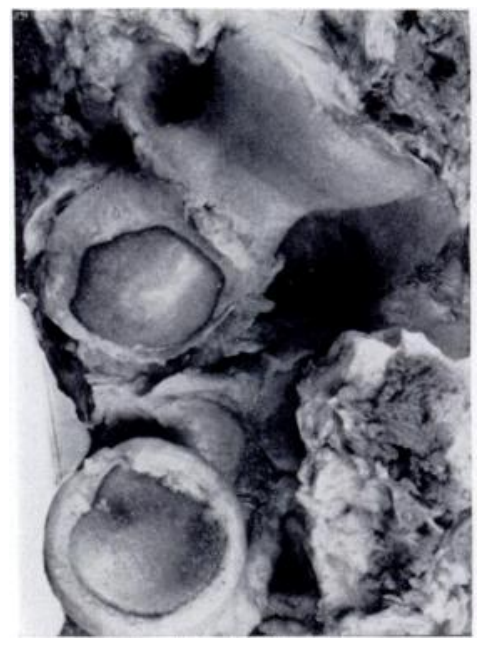

FIG. 9 


\section{MECHANICAL ENVIRONMENT OF THE CARTILAGE}

Such a pattern, or sequence of patterns, constantly repeated in many subjects, implies a mechanical explanation. What are the circumstances that dictate that in some areas the cartilage shall inevitably degenerate, early and progressively, and that in other areas the cartilage shall survive, equally inevitably, into old age? We will attempt an answer to these questions in terms of the nutritional and mechanical circumstances of the several areas of articular cartilage.

The radial head depicted in Figure 10 was prepared in the following way. It was excised from the intact elbow and then all the articular surfaces of the humerus and ulna were painted with a dye. The radial head was then replaced in the intact articulation and was put through a full range of pronation and supination in all positions from full flexion to full extension. Those parts of the radial head which came into contact with any of the articular surfaces of the other two bones picked up the dye. From this experiment we can describe the areas of articular cartilage under three headings.

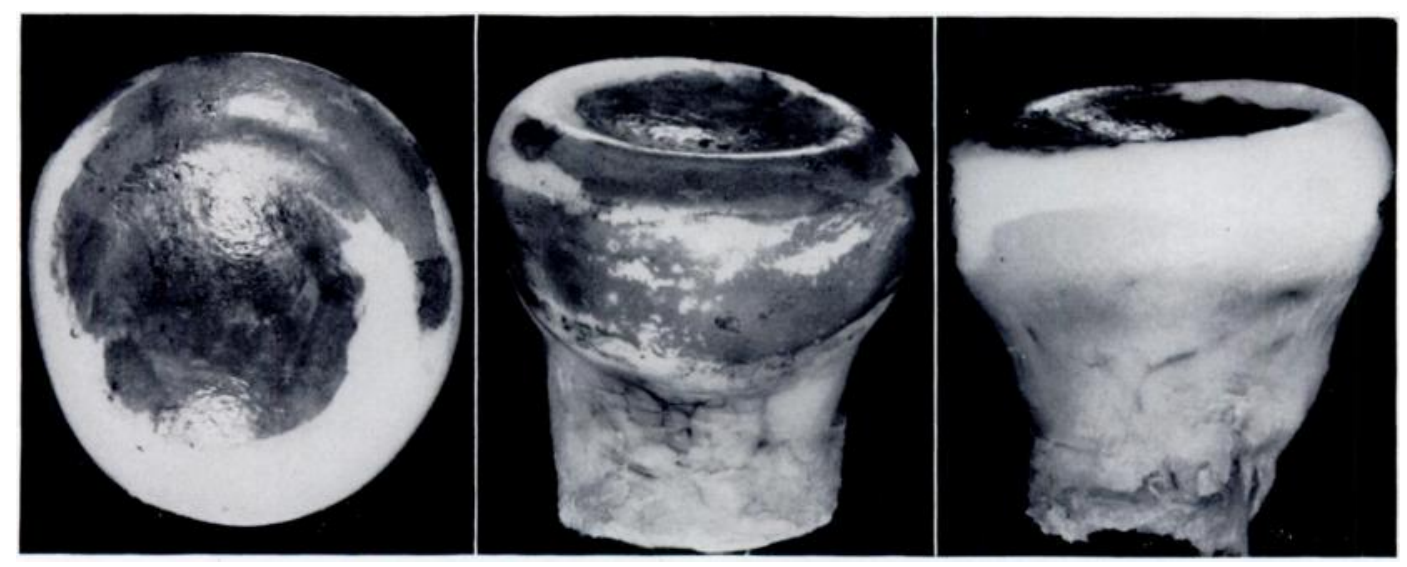

Fig. 10

Superior, medial and lateral views of the head of the radius with contact areas outlined by dye. For description see text.

Firstly, there are those parts that are usually in contact with opposed cartilage in all or nearly all positions of the joint. Thus the central depression on the radial head always articulates with the dome of the capitulum; the medial triangular facet is always in contact with the ulna.

Secondly, there are those areas that are never in contact with the opposed cartilage. Most of the rim of the radial head has failed to pick up any dye.

Thirdly, there is a small area, the posterior medial segment of the rim, which has picked up dye. This area, in common with the rest of the rim, in most positions of the joint fails to make contact with opposed cartilage. Unlike the rest of the rim it does touch the posterior part of the crest which separates the capitulum from the trochlea in one position only, which is full extension.

In contrast with the radio-humeral joint all the articular surfaces of the humero-ulnar articulation come into contact with opposed cartilage during some phase of movement. In the young adult joint in any position the contact is limited to two bands which extend across the articulation. In older people's joints the areas of contact become greater or, to put it another way, the joint becomes more congruous (Fig. 11). Having considered the contact areas of the articulation we must now go on to consider the nature of the movements which take place between opposed articular surfaces. Although the movements of the limb are complex the movements that actually occur between opposed cartilage surfaces are simple. They can move

VOl. 49 B, NO. 1, FEBRUARY 1967 


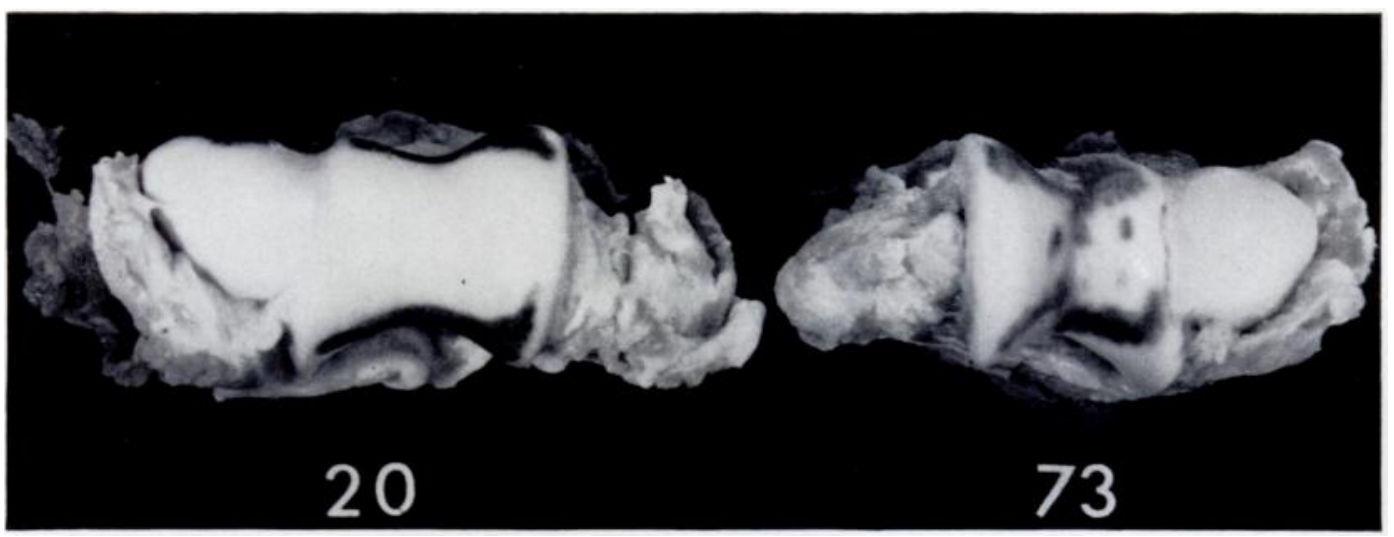

Fig. 11

Trochlear imprints of a young and an old joint compared in the mid-flexed position. In youth the trochlea touches the trochlear notch along two narrow bands but in old age the area of contact is more irregular and much more extensive.

on one another in two ways: they may either glide back and forth along a single pathway, or they may spin one upon another as they glide. In animal joints simple spinning about a fixed axis without some associated gliding does not occur.

\section{RELATIONSHIP OF JOINT MECHANICS TO THE PATTERN OF DEGENERATION}

Salter and McNeil (1965) observed that in joints with fixed deformity those areas of articular cartilage which were persistently in contact with synovial membrane underwent degenerative changes, and that in the absence of movement the synovial membrane became adherent to the adjacent cartilage. Our studies confirm the observations of Harrison, Schajowicz and Trueta (1953) that in normal joints those areas of cartilage which did not usually articulate with opposed cartilage inevitably became softened early in life. This degeneration in the physical state of the articular cartilage is called chondromalacia.

In most of the rim of the radial head the cartilage becomes chondromalacic early in life, but because of lack of contact with opposed articular surfaces this softened cartilage is subject only to slight wear and tear. However, the postero-medial part of the rim of the radial head, which also participates in the chondromalacic process, impinges forcibly against the posterior part of the crest separating the capitulum from the trochlea each time the elbow is fully extended, and is, because of the consequent trauma, the site of the constantly recurring ulcer.

In contrast, those areas of cartilage on the head which are always in contact with opposed articular cartilage survive well. The medial triangular facet which articulates with the ulna never wears out. On the other hand, the cartilage on the central depression of the head, while usually surviving into old age, does eventually wear away. If the nutritional environment of these two areas is similar, the " immortality" of the former must be due to the fact that it enjoys a less demanding mechanical environment than the latter.

We believe from our study of the elbow joint in particular, and of other articulations in general, that the quality of the movements that occur at a joint surface is the most significant factor in the mechanical environment of its articular cartilage. The opposed cartilages of the superior radio-ulnar joint glide on one another back and forth upon one set pathway. An irregularity of one surface of the single pathway joint will produce only a linear groove on the other, and when this groove is deep enough to accommodate the opposed ridge no further damage will take place. This occurs in the humero-ulnar articulation.

On the other hand, the opposed cartilages of the radio-humeral joint are required to spin upon one another in pronation and supination while at the same time they glide 
upon one another in flexion-extension. Any irregularity of either surface will under these conditions produce a devastating effect upon the other; the movement of the head of the radius on the capitulum could be likened to the movements of a pot scourer.

The observed pattern of the age changes in the elbow joint can therefore be explained on the basis of: 1) the nutritional environment; and 2) the mechanical environment of the variously affected areas of cartilage. We believe that something may be learned of the mechanical properties of cartilage in general from the consideration of its response to the mechanics of the elbow joint in particular.

\section{SUMMARY}

1. The age changes in the articular cartilage of the elbow joint are presented from a study of twenty-eight necropsy subjects aged eighteen to eighty-eight years. During early adult life those areas of cartilage which do not usually articulate with opposed cartilage always show some degree of chondromalacia.

2. Evidence is presented that the almost inevitable degeneration of the radio-humeral joint in old age is related to the combination of rotation and hinge movements that occur at that joint. This is in marked contrast with the relative immunity of the humero-ulnar articulation which has hinge movement only.

The photographs are the work of Mr David Drury of the Photographic Department of the Nuffield Orthopaedic Centre, to whom we express our thanks.

\section{REFERENCES}

Barnett, C. H., Davies, D. V., and MacConaill, M. A. (1961): Synovial Joints: Their Structure and Mechanics. London: Longman, Green and Co.

Bennett, G. A., WAINe, H., and BAUER, W. (1942): Changes in the Knee Joint at Various Ages with Particular Reference to the Nature and Development of Degenerative Joint Disease. New York: The Commonwealth Fund.

Eichelberger, L., Akeson, W. H., and Roma, M. (1958): Biochemical Studies of Articular Cartilage. I. Normal Values. Journal of Bone and Joint Surgery, 40-A, 142.

Екногм, R. (1956): Osteo-arthritis in the Knee-joint with Special References to the Weight-bearing in the Joint. Acta Morphologica Neerlando-Scandinavica, 1, 63.

Harrison, M. H. M., Schajowicz, F., and Trueta, J. (1953): Osteoarthritis of the Hip: A Study of the Nature and Evolution of the Disease. Journal of Bone and Joint Surgery, 35-B, 598.

Hirsch, C. (1944): A Contribution to the Pathogenesis of Chondromalacia of the Patella. Acta Chirurgica Scandinavica, Supplementum 83.

Little, K., Pimm, L. H., and Trueta, J. (1958): Osteoarthritis of the Hip. Journal of Bone and Joint Surgery, 40-B, 123.

Lloyd-RoberTs, G. C. (1955): Osteoarthritis of the Hip. Journal of Bone and Joint Surgery, 37-B, 8.

SAlter, R. B., and MCNeIL, R. (1965): Pathological Changes in Articular Cartilage Secondary to Persistent Jnint Deformity. Journal of Bone and Joint Surgery, 47-B, 185. 